\title{
Exercícios físicos como estratégia de prevenção e tratamento da obesidade: aspectos fisiológicos e metodológicos
}

\author{
Physical exercises as a strategy to prevent and to treat obesity: \\ physiological and methodological aspects
}

\author{
Ingrid B. F. Dias, Rafael A. Montenegro, Walace D. Monteiro*
}

\begin{abstract}
Resumo
A obesidade tem assumido proporções epidêmicas em vários países no mundo, incluindo o Brasil, onde também tem sido considerada um importante problema de saúde pública. A doença é caracterizada pelo acúmulo indevido de gordura em todos os sítios de depósito. Na etiologia da obesidade, encontram-se fatores cujas origens podem estar vinculadas a características genéticas, nutricionais, endócrinas, hipotalâmicas, farmacológicas, ambientais e comportamentais (principalmente a inatividade física), que se inter-relacionam e se potencializam mutuamente. Atualmente, o tecido adiposo tem sido reconhecido como um órgão multifuncional, produtor e secretor de vários peptídeos e proteínas bioativas, que estão envolvidas na inflamação e na resposta do sistema imune. De acordo com sua localização, o adipócito apresenta características metabólicas diferentes, sendo que o excesso de gordura, principalmente localizado na região abdominal, apresenta maior risco para o desenvolvimento de diabetes mellitus tipo 2 e de doença cardiovascular. Concomitantemente à obesidade, a inatividade física tem sido considerada um fator de risco independente para doença cardiovascular. Por isso, a prática sistemática de exercícios aeróbios e resistidos vem sendo recomendada como uma das principais intervenções não farmacológicas para prevenção e tratamento da obesidade e dos fatores de risco associados. Contudo, para que os exercícios possam promover benefícios, sua prescrição deve ser realizada com bases metodológicas consistentes. Desta forma, o presente artigo tem como objetivo revisar o papel dos exercícios aeróbios e resistidos na obesidade, bem como detalhar as diretrizes utilizadas no delineamento de sessões de treinamento físico direcionadas a indivíduos obesos. Para tanto, o texto foi organizado em quatro sessões. A primeira é dedicada aos efeitos dos exercícios resistidos na composição corporal, marcadores inflamatórios e fatores de risco associados à doença cardiovascular. Na segunda sessão, é abordada a atuação do exercício aeróbio no gasto calórico, bem como o papel da intensidade do exercício neste contexto. A terceira sessão é destinada às recomendações para a prescrição do exercício em obesos, destacando-se aí os exercícios resistidos e aeróbios. Nesta sessão, são detalhados os aspectos metodológicos que regem a estruturação das diferentes variáveis de prescrição do exercício. Por fim, são tecidas algumas considerações finais, sumarizando alguns aspectos detalhados na revisão.
\end{abstract}

Descritores: Obesidade; Exercício; Controle de risco.

\begin{abstract}
Obesity has assumed epidemic proportions in many countries worldwide, including Brazil, where it has also been considered as a major public health problem. The disease is characterized by an undesired accumulation of fat in all the deposit sites. The etiology of obesity has factors whose origins may be linked to genetic, nutritional, endocrine, hypothalamic, pharmacological, behavioral and environmental (mainly physical inactivity), which are interrelated and feed off of each other. Currently, adipose tissue has been recognized as a multifunctional organ that produces and secretes a number of bioactive peptides and proteins, which are involved in inflammation and immune response. According to its location, the adipocyte has different metabolic characteristics, and the fat excess, mainly located in the abdominal region shows a higher risk for developing type 2 diabetes mellitus and cardiovascular disease. Concomitant with obesity, physical inactivity has been considered an independent risk factor for cardiovascular disease. Therefore, the systematic practice of aerobic and resistance exercise has been recommended as a major non-pharmacological intervention for prevention and treatment of obesity and
\end{abstract}


its associated risk factors. However, to promote benefits exercise prescription must be made with consistent approaches. Thus, this article aims to review the role of aerobic and resistance exercises in obesity, as well as to detail guidelines used in the design of physical training sessions targeting obesity. Therefore, the text was organized in four sessions. The first one was devoted to the effects of resistance training on body composition, inflammatory markers and risk factors associated with cardiovascular disease. The second session was about the performance of aerobic exercise on energy expenditure and the role of exercise intensity in this context. The third session was designed to describe recommendations for exercise prescription in obesity, highlighting the aerobic and resistance exercises. This session details the methodological aspects that govern the structure of the different variables of the exercise prescription. Finally, conclusions were made summarizing some aspects described in this review.

Keywords: Obesity; Exercise; Risk management.

\section{Introdução}

A obesidade é uma doença crônica definida pelo excesso de gordura corporal, como resultado de um desequilíbrio energético influenciado por fatores cuja origem pode estar vinculada a características genéticas, nutricionais, endócrinas, hipotalâmicas, farmacológicas, ambientais e comportamentais (principalmente a inatividade física), que se inter-relacionam e se potencializam mutuamente. ${ }^{1}$ Na prática clínica, é comumente diagnosticada através do índice de massa corpórea (IMC), calculado através da razão entre o peso $(\mathrm{kg})$ e o quadrado da altura (m). Para utilização do IMC, deve-se assumir que ocorre uma distribuição igual de gordura no corpo inteiro do indivíduo. Contudo, esta análise não leva em conta a heterogeneidade da deposição regional de gordura, sendo esta um fator importante na correlação da obesidade com distúrbios metabólicos que levam a doenças cardiovasculares, diabetes mellitus do tipo 2 (DM2), dislipidemia, hipertensão, câncer, apneia do sono e síndrome metabólica. ${ }^{1}$

O excesso de gordura visceral acelera a mobilização dos ácidos graxos pelo sistema porta devido ao aumento de lipólise neste sítio. A elevada concentração de ácidos graxos livres no fígado e o excesso de gordura corporal estão associados à maior incidência de distúrbios metabólicos, hormonais, inflamatórios e cardiovasculares, como intolerância à glicose, dislipidemia, hiperinsulinemia, resistência à insulina e estresse oxidativo, com redução crônica da disponibilidade do óxido nítrico. ${ }^{2}$ Todos estes em conjunto implicarão em maior acometimento do sistema cardiovascular e impacto negativo sobre os órgãos-alvo, particularmente sobre o eixo cardiorrenal.

Atualmente, o tecido adiposo tem sido reconhecido como um órgão multifuncional, produtor e secretor de vários peptídeos e proteínas bioativas, denominadas adipocitocinas, que estão envolvidas na inflamação e na resposta do sistema imune. ${ }^{3}$ De acordo com sua localização, o adipócito apresenta características metabólicas diferentes, sendo que o excesso de gordura, principalmente localizado na região abdominal, apresenta maior risco para o desenvolvimento de DM2 e de doença cardiovascular (DCV). Quando comparado à gordura corporal total, o tecido adiposo visceral (TAV) apresenta maior correlação com os triglicerídeos (TG), pressão arterial (PA) sistólica, PA diastólica, e está associado com a resistência insulínica e inflamação de baixo grau. ${ }^{4}$ Logo, o excesso ou a distribuição desfavorável de gordura devem ser combatidos devido aos elevados riscos impostos à saúde.

A obesidade tem assumido proporções epidêmicas em vários países no mundo, incluindo o Brasil, no qual também tem sido considerada um importante problema de saúde pública. ${ }^{5}$ Concomitantemente à obesidade, a inatividade física tem sido considerada um fator de risco independente para DCV. Por isso, a prática sistemática de exercícios físicos vem sendo recomendada como uma das principais intervenções não farmacológicas, com o objetivo de prevenção primária e tratamento da obesidade e dos fatores de risco associados. ${ }^{6}$

Os exercícios físicos são recomendados como componente indispensável de um programa de controle, redução e prevenção do ganho de massa corporal. Para que tais objetivos sejam atingidos, a prática regular dos treinamentos aeróbio e com exercícios resistidos (ER) tem sido recomendada, devido às modificações favoráveis no gasto calórico, massa corporal magra e gordura corporal, concorrendo para o emagrecimento. ${ }^{6}$ Dese modo, o presente artigo tem como objetivo revisar a atuação dessas formas de treinamento na obesi- 
dade, bem como detalhar algumas diretrizes que podem ser usadas para prescrição de exercícios em indivíduos obesos.

\section{Exercícios resistidos e obesidade}

A inclusão dos ER como parte integrante de um programa de atividade física vem sendo recomendada por organizações de saúde como a American Heart Association. ${ }^{7}$ Embora essas recomendações sejam baseadas principalmente nos efeitos dos ER sobre a força muscular, estudos transversais têm demonstrado que a massa muscular está inversamente associada com todas as causas de mortalidade e prevalência da síndrome metabólica, independentemente dos níveis de aptidão cardiorrespiratória., ${ }^{8,9}$

Variáveis metodológicas como sobrecarga ou intensidade, duração e volume de treinamento, ordem dos exercícios, tempo de intervalo entre as séries, exercícios e sessões, número de séries e repetições, frequência semanal e velocidade de movimento estão envolvidas na elaboração de um programa de ER. Estas podem ser manipuladas de acordo com a especificidade do treinamento, objetivos e necessidades individuais. ${ }^{10}$ Tais variáveis podem desencadear diferentes respostas fisiológicas durante sua realização, tendo impacto direto na segurança cardiovascular no decorrer do treinamento.

Os ERs ainda são pouco estudados como parte integrante de um programa de exercícios para o tratamento e prevenção da obesidade. Tal fato pode ser explicado, em parte, pelo menor gasto energético e mobilização de gordura quando comparado ao exercício aeróbio (EA). No entanto, diferenças na resposta hormonal ao ER (potencial para a estimulação aguda da taxa metabólica e oxidação de gordura após a atividade) e de melhoria do balanço proteico muscular (potencial para aumentar o gasto energético total cronicamente) podem justificar a investigação dos benefícios desse tipo de treinamento na composição corporal. ${ }^{6}$

O treinamento aeróbio é considerado como uma modalidade de exercício eficaz para aumentar o gasto energético e o consumo de oxigênio pós-exercício (Excess Post-exercise Oxygen Consumption - EPOC), entretanto, estudos têm demonstrado que a prática de ER também pode exercer uma influência positiva sobre estas variáveis. Evidências sugerem que os ER possam desencadear um EPOC significativo para o controle do peso corporal e, adicionalmente, alguns estudos têm reportado um EPOC significativamente maior em resposta aos ER quando comparado ao EA."

Farinatti e colaboradores, ${ }^{12}$ por exemplo, investigaram os efeitos dos ERs realizados com diferentes intervalos de recuperação, utilizando séries múltiplas em pequenos e grandes grupamentos musculares sobre o consumo de oxigênio e gasto energético em homens saudáveis. Os autores demonstraram que o consumo de oxigênio durante e após uma sessão de ER realizada com número de repetições e sobrecarga similares pode ser influenciado pela massa muscular exercitada, assim como pelo intervalo de recuperação utilizado na sessão de treinamento. O consumo de oxigênio foi maior na sessão de ER composta por grandes grupamentos musculares quando comparado à sessão de ER executada com pequenos grupamentos musculares. Além disso, os autores também observaram que os intervalos de recuperação influenciaram a demanda metabólica no decorrer das séries.

De acordo com as diretrizes para prescrição de exercícios para a perda de peso e prevenção do reganho de peso corporal em adultos do ACSM (American College of Sports Medicine), ${ }^{13}$ até o momento, as evidências científicas existentes na literatura não suportam que a prática de ER seja eficaz para perda de peso, com ou sem restrição alimentar. No entanto, alguns estudos têm sugerido que a prática regular dos ERs pode ser eficaz na promoção da perda de peso em indivíduos obesos. ${ }^{14}$ Recentemente, em revisão sobre o potencial clínico dos ERs no tratamento da obesidade, Strasser e colaboradores ${ }^{15}$ demonstraram que a prática dos ERs esteve associada com uma diminuição da massa de gordura e um concomitante aumento da massa corporal magra e, portanto, com pouca ou nenhuma mudança efetiva no peso corporal.

Embora os efeitos dos ERs sobre a massa corporal total e a composição corporal possam ser modestos, a prática dos ERs tem sido associada com melhorias em fatores de risco cardiovascular na ausência de perda de peso significativa. Há ainda estudos que demonstram redução no colesterol total, no LDL-c (Low Density 
Lipoprotein cholesterol), nos TG e aumento no HDL-c (High-density Lipoprotein cholesterol), além de melhora na sensibilidade à insulina e redução nas concentrações de insulina plasmática glicose-estimulada. ${ }^{16}$

Recentemente, evidências têm sugerido que programas de ER supervisionados, desde que sejam bem elaborados, podem ser tão ou mais seguros do que muitos esportes de contato ou de alto impacto, principalmente para crianças e adolescentes. ${ }^{17}$ Dessa forma, o treinamento da força muscular foi incluído como um componente essencial de aptidão física nas recentes recomendações de jovens para a prática de atividade física. ${ }^{18}$ Alguns estudos realizados com crianças e adolescentes têm demonstrado alterações positivas em seus perfis metabólicos, independentemente da perda de peso ou até alterações na composição corporal. ${ }^{19-21}$ Benson e colaboradore ${ }^{19}$ realizaram uma revisão sistemática com objetivo de investigar o efeito dos ERs sobre o perfil metabólico de crianças e adolescentes, na qual foram incluídos 12 estudos de intervenção com ER. Os autores não observaram diferenças significativas para o IMC, composição corporal, colesterol total, LDL-c, HDL-c e TG. No entanto, foram demonstradas melhorias para a glicemia de jejum e sensibilidade à insulina, independentemente de mudanças na composição corporal.

Em outro estudo, Van der Heijden e colaboradores ${ }^{21}$ reportaram aumento na força muscular, massa corporal magra, sensibilidade à insulina e redução na taxa de produção de glicose após 12 semanas de intervenção exclusivamente com ER em adolescentes obesos. Em contraste, a mesma intervenção não foi capaz de promover mudanças na gordura total, visceral, hepática e intramiocelular dessa população.

Em recente revisão, Dietz e colaboradores ${ }^{20}$ investigaram o efeito da prática exclusiva de ER sobre a composição corporal e fatores de risco cardiovasculares. Dos seis estudos incluídos na revisão, quatro reportaram mudanças significativas na composição corporal, com aumento da massa magra e IMC e diminuição na massa de gordura. Três estudos analisaram o efeito da prática dos ER sobre os fatores de risco cardiovascular, e apenas um demonstrou redução significativa na PA sistólica.
Os dados relacionados ao efeito de um treinamento exclusivo com ER em crianças e adolescentes obesos ainda permanecem inconclusivos. Estudos randomizados controlados ainda são insuficientes para definir a prescrição de um programa de ER com eficácia clínica para prevenção e tratamento de doenças cardiometabólicas nessa população.

\section{Exercícios aeróbios e obesidade}

Os benefícios da prática de atividade física ou engajamento em programas de exercício físico, acarretando mudanças no estilo de vida sedentário, estão bem documentados na literatura, constituindo importante intervenção no tratamento da obesidade.22 Outro fator primordial na tentativa de reversão do quadro da obesidade é a modificação dos padrões alimentares. Portanto, é fácil de pensar que dietas hipocalóricas parecem ser a melhor medida adotada para quem deseja perder peso. No entanto, a associação de ingestão alimentar balanceada com aumento relevante do dispêndio energético diário total parece ser ainda mais benéfica, pois além de uma redução da massa gorda, há uma manutenção da massa magra. ${ }^{23}$

O exercício aeróbio vem sendo amplamente utilizado em programas de emagrecimento como forma de exercício físico mais eficaz para perda de massa corporal, principalmente da massa de gordura. Isto se justifica pelo aumento do deficit energético gerado por tal exercício, tanto durante como após o término do mesmo. O aumentado consumo de oxigênio mesmo após o término do esforço (EPOC) é um dos fatores positivos do exercício aeróbio em relação à perda e/ou manutenção do peso corporal.

Imediatamente após o término do exercício aeróbio, um EPOC rápido parece acontecer decorrente da ressíntese de ATP-CP, da remoção do lactato sanguíneo, do aumento na temperatura corporal e do metabolismo lipídico. Este consumo de oxigênio parece ainda permanecer aumentado por horas (EPOC lento) decorrente principalmente do ciclo dos triglicerídeos/ácidos graxos. Porém, a magnitude e duração do EPOC parecem ser relacionadas linearmente com o tempo e exponencialmente com a intensidade do esforço. ${ }^{24}$ Em resumo, exercícios aeróbios de alta intensidade e longa duração parecem promover 
um maior gasto energético e, além disso, podem causar aumento da aptidão cardiorrespiratória ( $\mathrm{VO}_{2}$ máx - consumo máximo de oxigênio).

Outro fator positivo do EA sobre a obesidade se dá pela estreita relação entre o impacto deste sobre a ingestão alimentar e queima de gordura, pois o trabalho corporal requer energia fornecida pelos nutrientes para que haja restauração dos estoques de combustível, com o propósito da continuidade do funcionamento das funções corporais. ${ }^{25}$

Whybrow e colaboradores ${ }^{26}$ analisaram o efeito do exercício aeróbio em diferentes intensidades verificadas em grupos que não realizaram nenhum exercício (grupo controle) e grupos que realizaram exercícios com moderada e elevada intensidades, sobre o apetite, ingestão alimentar, dispêndio e balanço energético em homens e mulheres. Os autores constataram que o dispêndio energético gerado pelo exercício aeróbio começa a ser compensado ou contrabalanceado pela ingestão alimentar, num curso de uma a duas semanas. Nesse sentido, mais recentemente, King e colaboradores ${ }^{27}$ esclareceram que o efeito do EA sobre a regulação do apetite envolveria um aumento em todos os sinais orexígenos e um concomitante aumento na eficiência de sinais de saciedade. No entanto, os mesmos autores alegaram que estes processos não operam com a mesma força e eficácia em todos os indivíduos praticantes de exercícios. Juntas, as forças destes dois processos podem determinar se indivíduos perderão peso com a prática de exercício, ou se o peso corporal será mantido através do efeito compensatório do aumento da ingestão alimentar após a sessão de exercício aeróbio.

Em relação às respostas compensatórias ao deficit energético gerado pelo exercício aeróbio, King e colaboradores ${ }^{28}$ sugeriram que a influência das respostas compensatórias metabólicas (respostas automáticas ou obrigatórias, como a taxa metabólica de repouso e o dispêndio energético gerado por outras atividades fisiológicas) e comportamentais (respostas voluntárias ou facultativas, como a ingestão alimentar) seria um ponto-chave no tocante à barreira para perda de peso induzida pela prática de exercício aeróbio. As respostas compensatórias comportamentais possuiriam maior poder influenciador sobre o balanço energético do que as respostas metabólicas.
Portanto, as contribuições do exercício físico nas perturbações ao balanço energético são menos expressivas do que as contribuições dadas por meio da ingestão alimentar. ${ }^{29}$

No tocante às variações do EA destinadas à perda ou manutenção do peso corporal, o EA intervalado de alta intensidade (High-intensity Interval Training - HIIT) tem ganhado bastante notoriedade nos últimos anos. Os HIITs são uma forma aperfeiçoada de treinamento intervalado que utilizam estratégias de alternância entre curtos períodos de exercícios intensos com períodos de recuperação ativa. Esta modalidade de treinamento físico, quando comparada ao exercício aeróbio contínuo tradicional, vem apresentando satisfatórios resultados sobre o desempenho físico, bem como um aumentado metabolismo de glicose, ao mesmo tempo que favorece a oxidação lipídica e consequentemente o rápido emagrecimento. ${ }^{30,31}$ Além disso, Gremeaux e colaboradores $^{32}$ relataram que os HIITs vêm demonstrando ser uma forma segura, eficiente e bem tolerada por indivíduos normoponderais e obesos, com taxas de $97 \%$ de aderência e nenhum evento adverso contabilizado durante o treinamento físico nestas populações.

A partir das evidências acima expostas, parece que o efeito de sessões agudas de exercícios aeróbios sobre o gasto calórico e emagrecimento depende extremamente da intensidade do exercício empregado. Exercícios aeróbios com intensidades de moderada a extenuante ( $>60 \% \mathrm{VO}_{2}$ máx) parecem ser mais eficazes no tocante ao efeito supressor da ingestão alimentar, bem como sobre a magnitude do dispêndio energético gerado durante e após o exercício. Além disso, os exercícios intervalados de alta intensidade parecem ser uma excelente estratégia para otimização dos resultados em um programa de emagrecimento.

\section{Recomendações para a prescrição do exercício em obesos}

Como já destacado, sobrepeso e obesidade estão relacionados a diversas doenças crônicas incluindo-se as doenças cardiovasculares, o DM2, diversas formas de câncer, além de numerosos problemas musculoesqueléticos. Associados a estes, somam-se ainda problemas de ordem psi- 
cológica como a depressão e a ansiedade, entre outros. ${ }^{13}$

Se por um lado diversas doenças estão associadas ao excesso de peso, a prática regular do exercício está relacionada à redução ou controle desta condição, e consequentemente, à promoção da saúde. Contudo, para que um programa de exercícios exerça um papel positivo no controle do peso corporal, ele deve ser prescrito com bases metodológicas consistentes, envolvendo os seguintes componentes: duração do esforço, intensidade do esforço, frequência semanal das atividades, tipo de atividade executada e forma de progressão do treinamento. Estes componentes devem ser organizados de modo a provocar um balanço energético negativo e uma melhora na composição corporal dos praticantes, incluindo-se a redução da gordura corporal e o aumento da massa corporal magra. ${ }^{23}$

Como destacado pelo ACSM, ${ }^{13}$ a quantidade de atividade física que poderá ser necessária para preservar a perda de peso e prevenir a recuperação do mesmo deve envolver as seguintes considerações:

1 - a modalidade de atividade deve ser preferencialmente aeróbia, que envolva grandes grupamentos musculares, seja simples e, preferencialmente, possa ser mantida durante um maior período. Nesse caso, atividades como a caminhada, o ciclismo estacionário ou ao ar livre e a corrida são preconizadas. Outras atividades como patinação, natação e remo podem ser realizadas. Contudo, por exigirem maior técnica para sua execução, necessitam de maior período de tempo para o aprendizado, o que pode desencorajar os praticantes. $\mathrm{O}$ acréscimo do trabalho contra resistência também deve ser encorajado devido aos seus efeitos positivos nas estruturas da composição corporal (gordura corporal e principalmente massa corporal magra) e na aptidão física geral, destacando-se aí a força e a resistência muscular;

2 - a frequência semanal de treinamento deve ser de cinco a sete dias. Em alguns casos, pode-se iniciar a prescrição com uma frequência de três dias e, com a evolução do condicionamento, evoluir para sete dias na semana. É importante destacar que a frequência do treinamento está estritamente relacionada à duração e intensidade do esforço. O somatório desses aspectos, associado às respostas individuais do praticante, é que norteou o delineamento da frequência semanal trabalhada;

3 - inicialmente, levando-se em conta que os indivíduos geralmente não são bem condicionados, a duração das atividades deve ser de 30 minutos. Com o aprimoramento do condicionamento, deve-se buscar atingir 60 minutos de atividades físicas diárias. Os adultos com sobrepeso e obesidade podem acumular essa quantidade de atividade em múltiplas sessões de dez minutos de duração ou através de aumentos em outras formas de atividades relacionadas ao estilo de vida com intensidade moderada. Em alguns casos, para que ocorram perdas mais significativas do peso corporal, programas com duração de 60 a 90 minutos ao dia podem ser realizados;

4 - para indivíduos mal condicionados que estão iniciando um programa de exercícios, a intensidade do esforço deve ser baixa ou moderada. Nesse sentido, preconiza-se adotar 40 a $60 \%$ do consumo de oxigênio de reserva $\left(\mathrm{VO}_{2} \mathrm{R}\right)$ ou da frequência cardíaca de reserva (FCR). A progressão para uma intensidade mais elevada deve ser alvo com a evolução do condicionamento. Nesse caso, deve-se trabalhar em uma faixa de esforço entre 50 a $75 \%$ do $\mathrm{VO}_{2} \mathrm{R}$ ou da FCR. A percepção do esforço é outro indicador de intensidade que pode ser usado para quantificar a intensidade do exercício aeróbio, devido a sua praticidade e relação com o $\mathrm{VO}_{2} \mathrm{R}$ e a FCR. Nesse caso, deve-se trabalhar numa faixa com variação de quatro a sete em uma escala de dez pontos. ${ }^{33}$ É importante destacar que o sucesso da aplicação da Escala de Borg está relacionado à ambientação do indivíduo com sua utilização para uma dada modalidade de atividade (por exemplo: caminhada, bicicleta etc). Por isso, uma pessoa treinada em usar a escala no cicloergômetro, não obrigatoriamente a usará corretamente na corrida ou na natação;

5 - a progressão do treinamento é um aspecto muito individual e depende do somatório 
envolvendo frequência, duração e intensidade do esforço. Como regra básica, pode-se assumir que um indivíduo, ao perceber que o esforço imposto por uma sessão de exercícios não está forte, pode aumentar ligeiramente este esforço na sessão seguinte e assim por diante. Outra forma bastante eficiente de se trabalhar para aumentar a sobrecarga de treinamento é alternar sessões de intensidade mais elevada e menor duração com sessões de intensidade menos elevada e maior duração, ao longo da semana. O importante é progredir na frequência, duração e intensidade do esforço conforme o praticante for se adaptando às sessões anteriores. Como destacado por McArdle e colaboradores,$^{23}$ progressões excessivamente rápidas revelam-se contraproducentes, pois a maioria dos indivíduos com excesso de peso se opõe a esse tipo de progressão no início. Além disso, progressões muito acentuadas podem tornar o exercício menos prazeroso, o que pode levar à redução na adesão aos programas de exercícios. ${ }^{13}$

No que diz respeito ao trabalho contra resistência, as variáveis de prescrição do treinamento são as seguintes: seleção dos exercícios, número de séries, número de repetições por série, cargas utilizadas, intervalos entre séries e exercícios, frequência semanal e forma de condução do treinamento. Assim como no trabalho aeróbio, o resultado alcançado depende da forma pela qual as variáveis serão combinadas. Logo, os objetivos com a prescrição e o condicionamento do praticante determinarão a melhor combinação. Antes de falar especificamente de cada variável de prescrição é importante ressaltar que as normas de prescrição existentes são preconizadas para indivíduos que desejam aprimorar a aptidão física geral e, por isso, também se aplicam aos indivíduos obesos ou com sobrepeso.

$\mathrm{Na}$ seleção dos exercícios, geralmente são executados aqueles que recrutam os principais grupamentos musculares acionados nas atividades cotidianas. Em geral, de oito a 12 exercícios são executados para grupamentos musculares variados. ${ }^{13}$ Dentre os mais utilizados, destacamos o desenvolvimento supino, a puxada pela frente, as remadas altas e ao peito, o desenvolvimento pela frente, as cadeiras extensora e flexora, adutora e abdutora, o leg press e os abdominais. Exercícios mais específicos envolvendo menores grupamentos musculares como roscas, bíceps e tríceps e a flexão plantar também são muito usados. Uma descrição desses exercícios, bem como alguns cuidados a serem adotados na execução dos mesmos podem ser vistos em literatura complementar. ${ }^{34}$ Como os exercícios contra resistência geralmente são realizados na mesma sessão em que os exercícios aeróbios, a quantidade de exercícios realizada poderá sofrer influência das características e do tempo destinado à prescrição do treinamento aeróbio.

Quanto ao número de séries, geralmente são conduzidas de duas a quatro. Essas séries poderão ser derivadas do mesmo exercício ou de uma combinação de exercícios que incidam no mesmo grupamento muscular. ${ }^{13}$ Indivíduos mal condicionados, em alguns casos, podem começar o programa de treinamento executando de uma a duas séries, mas na prática, isso não é muito comum. As repetições máximas em cada série variam de 8 a 12. Essa faixa é amplamente empregada devido às possibilidades de obtenção da força e resistência muscular. Contudo, ressaltamos que em uma fase de aprendizado do exercício, o conceito de repetição máxima não necessita ser enfatizado. Nesse caso, repetições submáximas podem ser realizadas e, a partir do momento em que o aprendizado for efetivado, o praticante passará a trabalhar adotando repetições máximas nas séries.

As cargas usadas dependem do número de repetições máximas realizadas e, geralmente, situam-se entre 60 e $80 \%$ de uma repetição máxima (1 RM). Os intervalos entre séries e exercícios, comumente, variam de dois a três minutos e devem ser aplicados de modo a promover uma adequada recuperação para realização das séries subsequentes com as repetições propostas. ${ }^{34}$ Logo, uma variação mais ou menos acentuada na duração dos intervalos pode ser verificada nas primeiras ou últimas séries realizadas em um dado exercício, assim como em exercícios para distintos grupamentos musculares.

No que concerne à forma de condução, o treinamento pode ser realizado através de vários sistemas. Monteiro e Farinatti ${ }^{34}$ descrevem certos aspectos metodológicos da aplicação de alguns sistemas, cujas principais características são des- 
critas a seguir. Um dos sistemas mais tradicionais consiste em realizar os exercícios na forma de circuito. Nesse sistema, os exercícios são realizados sequencialmente, com pouco intervalo entre cada um (geralmente 30 segundos a 1 minuto). Em alguns casos, quando os grupamentos musculares são variados de um exercício para o outro, pode-se realizar a sequência sem intervalos. Contudo, no caso dos indivíduos obesos ou com sobrepeso, seria prudente aplicar ao menos um pequeno intervalo entre os exercícios para evitar a fadiga e não desmotivar os praticantes. Outra forma de trabalho bastante usual consiste no sistema de séries múltiplas, no qual ao menos três séries são realizadas para cada exercício. A sequência de aplicação dos exercícios nesse sistema pode ser bem variada. Podem ser realizadas séries em sequência para um mesmo exercício (ou exercícios distintos para um mesmo grupamento muscular), com intervalos de dois a três minutos. Quando essa forma de trabalho é aplicada, intervalos de dois a três minutos devem ser executados para evitar a fadiga na musculatura exercitada. $\mathrm{Na}$ realidade, uma boa estratégia para estabelecer os intervalos entre séries e exercícios consiste na utilização da percepção do esforço do praticante. Quando ele se sentir apto, uma nova série será então realizada. Alternar os segmentos também é uma boa estratégia de trabalho para os praticantes que executam o sistema de séries múltiplas, notadamente os obesos que iniciam o treinamento ou aqueles que não são bem condicionados. Nesse caso, pode-se conduzir o trabalho de forma alternada por segmento corporal (por exemplo: primeiro exercícios para braços e depois exercícios para pernas ou abdômen), ou agonista-antagonista (por exemplo: primeiro exercício para o quadríceps e depois para os músculos posteriores da coxa). Os intervalos entre séries e exercícios podem ser de dois a três minutos, mas, como já dito, isso pode ficar a cargo do praticante em função da percepção do esforço após a realização de cada série.

Como descrito, a perda de peso envolve um aspecto multifatorial, cujas recomendações extrapolam a redução no aporte calórico e o aumento no gasto energético. É importante que recomendações comportamentais sejam adicionadas para o sucesso no programa de exercícios. Com esse propósito, o ACSM $^{13}$ propôs algumas recomendações:
- incorporar oportunidades para melhorar a comunicação entre os profissionais da assistência de saúde, os nutricionistas, os profissionais do exercício e as pessoas com sobrepeso e obesidade após o período inicial de perda de peso;

- ter como alvo uma mudança nos comportamentos alimentar e relacionados ao exercício, pois as mudanças sustentadas em ambos resultam em uma perda de peso significativa em longo prazo;

- ter como alvo uma redução na ingestão de 500 a $1.000 \mathrm{kcal} /$ dia para conseguir a perda de peso. Essa redução deve ser combinada com uma diminuição de 30\% na gordura ingerida;

- ter como alvo um aumento progressivo para um mínimo de $150 \mathrm{~min} / \mathrm{semana}$ de atividade física de intensidade moderada para aprimorar os benefícios de saúde e aptidão física para os adultos com sobrepeso e obesos;

- progredir para quantidades mais altas de exercício (de 200 a $300 \mathrm{~min} / \mathrm{semana}$ ou maior ou igual a $2.000 \mathrm{kcal} / \mathrm{semana}$ ) de atividade física para promover o controle do peso em longo prazo;

- incorporar as estratégias de modificação comportamental, a fim de facilitar a adoção e a manutenção das mudanças desejadas no comportamento.

\section{Conclusões}

O exercício físico tem sido destacado como importante elemento na abordagem não farmacológica para o tratamento e prevenção da obesidade. Diversos estudos têm demonstrado que a prática sistemática do exercício atua positivamente na redução do peso corporal e nos fatores de risco associados. Tanto os exercícios aeróbios quanto os resistidos vêm sendo apontados como elementos fundamentais em um programa de exercício direcionado ao controle do peso corporal. Contudo, para que os exercícios possam promover benefícios, sua prescrição deve ser realizada com bases metodológicas consistentes. No caso dos indivíduos obesos ou com 
sobrepeso, as normas de prescrição do exercício não são muito diferentes em relação à utilizada em indivíduos eutróficos. As diferenças recaem nos cuidados a serem adotados, visto as comorbidades associadas à obesidade. Em geral, a prescrição do exercício envolve a manipulação de variáveis, incluindo-se a modalidade, a frequência semanal, o volume e a intensidade do esforço, bem como a forma de progressão do treinamento. A estruturação das variáveis deve ser específica para cada indivíduo, levando-se em conta seu estado clínico e de treinamento. Além disso, deve-se considerar as respostas apresentadas ao longo do treinamento. Seguindo esses passos, o exercício proporcionará efeitos positivos não somente no controle do peso corporal, mas também na promoção da saúde.

\section{Referências}

1. Godoy-Matos AF. Síndrome Metabólica. $1^{\mathrm{a}}$ ed. São Paulo: Editora Atheneu; 2005. 356 p.

2. Rutkowski JM, Davis KE, Scherer PE. Mechanisms of obesity and related pathologies: the macro- and microcirculation of adipose tissue. FEBS J. 2009 Oct;276(20):5738-46. http://dx.doi.org/10.1111 /j.1742-4658.2009.07303.

3. Hauner $\mathrm{H}$. The new concept of adipose tissue function. Physiol Behav. 2004 Dec;83(4):653-8. http:// dx.doi.org/10.1016/j.physbeh.2004.09.016.

4. Nieves DJ, Cnop M, Retzlaff B, Walden CE, Brunzell JD, Knopp RH, et al. The atherogenic lipoprotein profile associated with obesity and insulin resistance is largely attributable to intra-abdominal fat. Diabetes. 2003 Jan;52(1):172-9.

5. Ministério da Saúde. VIGITEL Brasil 2009: vigilância de fatores de risco e proteção para doenças crônicas por inquérito telefônico. Brasília, DF: Ministério da Saúde; 2010.

6. Donnelly JE, Blair SN, Jakicic JM, Manore MM, Rankin JW, Smith BK, et al. American College of Sports Medicine Position Stand. Appropriate physical activity intervention strategies for weight loss and prevention of weight regain for adults. Med Sci Sports Exerc. 2009 Feb;41(2):459-71. http://dx.doi.org/10.2337/diabetes.52.1.172.

7. Pollock ML, Franklin BA, Balady GJ, Chaitman BL, Fleg JL, Fletcher B, et al. AHA Science Advisory. Resistance exercise in individuals with and without cardiovascular disease: benefits, rationale, safety, and prescription: An advisory from the Committee on Exercise, Rehabilitation, and Prevention, Council on Clinical Cardiology, American Heart Association;
Position paper endorsed by the American College of Sports Medicine. Circulation. 2000 Feb;101(7):82833. http://dx.doi.org/10.1161/01.CIR.101.7.828.

8. Fitzgerald SJ, Barlow CE, Kampert JB, Morrow Jr. JR, Jackson AW, Blair SN. Muscular fitness and allcause mortality: prospective observations. J Phys Act Health. 2004;1:7-18.

9. Jurca R, Lamonte MJ, Barlow CE, Kampert JB, Church TS, Blair SN, et al. Association of muscular strength with incidence of metabolic syndrome in men. Med Sci Sports Exerc. 2005 Nov;37(11):1849-55. http:// dx.doi.org/10.1249/01.mss.0000175865.17614.74.

10. Kraemer WJ, Ratamess NA. Fundamentals of resistance training: progression and exercise prescription. Med Sci Sports Exerc. 2004 Apr;36(4):674-88.

11. Poehlman ET, Dvorak RV, DeNino WF, Brochu M, Ades PA. Effects of resistance training and endurance training on insulin sensitivity in nonobese, young women: a controlled randomized trial. J Clin Endocrinol Metab. 2000 Jul;85(7):2463-8. http://dx. doi.org/10.1210/jc.85.7.2463.

12. Farinatti PT, Castinheiras Neto AG. The effect of between-set rest intervals on the oxygen uptake during and after resistance exercise sessions performed with large- and small-muscle mass. J Strength Cond Res. 2011 Nov;25(11):3181-90. http://dx.doi.org/10.1519/ JSC.0b013e318212e415.

13. ACSM. Diretrizes do American College of Sports Medicine para os testes de esforço e sua prescrição. $8^{a}$ ed. Rio de Janeiro: Guanabara Koogan; 2010. 272 p.

14. Rice B, Janssen I, Hudson R, Ross R. Effects of aerobic or resistance exercise and/or diet on glucose tolerance and plasma insulin levels in obese men. Diabetes Care. 1999 May;22(5):684-91.

15. Strasser B, Schobersberger W. Evidence for resistance training as a treatment therapy in obesity. J Obes. 2011;2011.pii:482564. http://dx.doi. org/10.1155/2011/482564.

16. Fenkci S, Sarsan A, Rota S, Ardic F. Effects of resistance or aerobic exercises on metabolic parameters in obese women who are not on a diet. Adv Ther. 2006 May-Jun;23(3):404-13.

17. Faigenbaum AD. Strength training for children and adolescents. Clin Sports Med. 2000 Oct;19(4):593619.

18. Strong WB, Malina RM, Blimkie CJ, Daniels SR, Dishman RK, Gutin B, et al. Evidence based physical activity for school-age youth. J Pediatr. 2005 Jun;146(6):732-7. http://dx.doi.org/10.1016/j. jpeds.2005.01.055.

19. Benson AC, Torode ME, Fiatarone Singh MA. Effects of resistance training on metabolic fitness in children and adolescents: a systematic review. Obes Rev. 
2008 Jan;9(1):43-66. http://dx.doi.org/10.1111/j.1467789X.2007.00388.x.

20. Dietz P, Hoffmann S, Lachtermann E, Simon P. Influence of exclusive resistance training on body composition and cardiovascular risk factors in overweight or obese children: a systematic review. Obes Facts. 2012 Jul;5(4):546-60. http://dx.doi. org/10.1159/000341560.

21. Van der Heijden GJ, Wang ZJ, Chu Z, Toffolo G, Manesso E, Sauer PJ, et al. Strength exercise improves muscle mass and hepatic insulin sensitivity in obese youth. Med Sci Sports Exerc. 2010 Nov;42(11):197380. http://dx.doi.org/10.1249/MSS.0b013e3181df16d9.

22. Jakicic JM, Otto AD. Physical activity considerations for the treatment and prevention of obesity. Am J Clin Nutr. 2005 Jul;82(1 Suppl):226S-9S

23. McArdle WD, Katch Fl, Katch VL. Fisiologia do Exercício - Energia, Nutrição e Desempenho Humano. $7^{\mathrm{a}}$ ed. Rio de Janeiro: Guanabara Koogan; 2011. 1172 p.

24. Lima-Silva AE, Pires FO, Bertuzzi R. Excesso de oxigênio consumido pós-esforço: possíveis mecanismos fisiológicos. Rev Educ Fís/UEM. 2010 Set;21(3):563-75. http://dx.doi.org/10.4025/ reveducfis.v21i3.6283

25. Melzer K, Kayser B, Saris WH, Pichard C. Effects of physical activity on food intake. Clin Nutr. 2005 Dec;24(6):885-95. http://dx.doi.org/10.1016/j. clnu.2005.06.003

26. Whybrow S, Hughes DA, Ritz P, Johnstone AM, Horgan Gw, King N, et al. The effect of an incremental increase in exercise on appetite, eating behaviour and energy balance in lean men and women feeding ad libitum. Br J Nutr. 2008 Nov;100(5):1109-15. http:// dx.doi.org/10.1017/S0007114508968240

27. King NA, Caudwell PP, Hopkins M, Stubbs JR, Naslund E, Blundell JE. Dual-process action of exercise on appetite control: increase in orexigenic drive but improvement in meal-induced satiety.
Am J Clin Nutr. 2009 Oct;90(4):921-7. http://dx.doi. org/10.3945/ajcn.2009.27706

28. King NA, Hester J, Gately PJ. The effect of a medium-term activity- and diet-induced energy deficit on subjective appetite sensations in obese children. Int J Obes (Lond). 2007 Feb;31(2):334-9. http://dx.doi. org/10.1038/sj.ijo.0803391

29. Blundell JE, Finlayson G. Is susceptibility to weight gain characterized by homeostatic or hedonic risk factors for overconsumption? Physiol Behav. 2004 Aug;82(1):21-5. http://dx.doi.org/10.1016/j.physbeh.2004.04.021

30. Perry CG, Heigenhauser GJ, Bonen A, Spriet LL. High-intensity aerobic interval training increases fat and carbohydrate metabolic capacities in human skeletal muscle. Appl Physiol Nutr Metab. 2008 Dec;33(6):1112-23. http://dx.doi.org/10.1139/H08-097

31. Talanian JL, Galloway SD, Heigenhauser GJ, Bonen A, Spriet LL. Two weeks of high-intensity aerobic interval training increases the capacity for fat oxidation during exercise in women. J Appl Physiol. 2007 April;102(4):1439-47. http://dx.doi.org/10.1152/ japplphysiol.01098.2006.

32. Gremeaux V, Drigny J, Nigam A, Juneau M, Guilbeault $\mathrm{V}$, Latour $\mathrm{E}$, et al. Long-term lifestyle intervention with optimized high-intensity interval training improves body composition, cardiometabolic risk, and exercise parameters in patients with abdominal obesity. Am J Phys Med Rehabil. 2012 Nov;91(11):941-50. http://dx.doi.org/10.1097/PHM.0b013e3182643ce0.

33. Borg G. Borg's Perceived Exertion and Pain Scales. Champaign: Human Kinectics; 1998. 104 p.

34. Monteiro W, Farinatti PTV. Aspectos fisiológicos e metodológicos da prescrição do exercício para idosos: força e flexibilidade. In: Farinatti PTV, editor. Envelhecimento, promoção da saúde e exercício: bases teóricas e metodológicas. São Paulo: Manole, 2008. p.131-61.

$\begin{array}{ll}\text { Recebido: } & \text { 04/09/2013. } \\ \text { Revisado: } & \text { 26/11/2013. } \\ \text { Aprovado: } & \text { 04/02/2014. }\end{array}$

